\title{
Mejora del proceso de atención de incendios para incrementar la calidad del servicio brindado por la Compañía de Bomberos Voluntarios del Perú B 107 - Chimbote 2017
}

\section{Improvement of the fire assistance process to increase the quality of the service provided by the Volunteer Firefighters of Peru B 107 - Chimbote 2017}

\section{Melhoria do processo de assistência incêndio para aumentar a qualidade do serviço prestado pelos Bombeiros Voluntários do Peru B 107 - Chimbote 2017}

Mariby Karolay Untul Mariluz ${ }^{(1)}$; Walter Estela Tamay ${ }^{(2)}$; Lourdes Jossefyne Esquivel Paredes ${ }^{(2)}$

Fecha de recepción: 07 de noviembre de 2017 Fecha de aprobación: 28 de mayo de 2018

\section{Resumen}

La presente investigación tuvo como finalidad implementar una mejora en el proceso de atención de incendios para incrementar la calidad del servicio brindado por la Compañía de Bomberos Voluntarios del Perú B 107 - Chimbote 2017. Para ello se utilizó un diseño experimental del tipo pre experimental con pre test y post test, tomando como población a todos los procesos de atención de la compañía y como muestra al proceso de atención de incendios, aplicando un muestreo no probabilístico por conveniencia. El diagnóstico inicial mostró un total de ocho operaciones, una inspección, una demora y una transporte; de la misma manera se calculó un tiempo promedio para la atención de incendios de 54.62 minutos, un tiempo normal de 64,99 minutos y un tiempo estándar de 106,59 minutos. Asimismo la aplicación del cuestionario Servqual indicó que las dimensiones de la calidad del servicio se encontraban en un nivel medio. Posteriormente, a través de un diagrama causa - efecto, se determinó las mejoras necesarias en los métodos de trabajo optimizando el tiempo promedio hasta reducirlo en 33,72 minutos, el tiempo normal en 40,12 minutos y el tiempo estándar en 65,80 minutos representando una disminución del $61,74 \%$. Finalmente, se concluyó que la calidad del servicio mejoró en 26,73 puntos según la escala del Servqual, dicha diferencia fue validada estadísticamente con una prueba t de Student utilizando un intervalo de confianza del $95 \%$ y una significancia experimental de 0,026 .

Palabras clave: calidad del servicio, Servqual, mejora de procesos, estudio de tiempos

\begin{abstract}
The purpose of this research was to implement an improvement in the firefighting process to increase the quality of service provided by the Peruvian Volunteer Fire Company B 107 - Chimbote 2017. For this purpose, an experimental design of the pre-experimental type with pre test and post test, taking as a population all the company's attention processes and as a sample to the fire care process, applying a non-probabilistic sampling for convenience. The initial diagnosis showed a total of eight operations, one inspection, one delay and one transport; in the same way an average time for fire attention of 54,62 minutes was calculated, a normal time of 64,99 minutes and a standard time of 106,59 minutes; Likewise, the application of the Servqual questionnaire indicated that the dimensions of service quality were at a medium level. Subsequently, through a cause - effect diagram, the necessary improvements

\footnotetext{
${ }^{1}$ Autora corresponsal: Mariby Untul. Universidad César Vallejo. Chimbote, Perú; Facultad de Ingeniería; Email: mariby275@gmail.com

${ }^{2}$. Universidad César Vallejo. Chimbote, Perú.; Facultad de Ingeniería.
} 
in work methods were determined by optimizing the average time to reduce it in 33,72 minutes, the normal time in 40,12 minutes and the standard time in 65,80 minutes representing a decrease of $61,74 \%$. Finally, it was concluded that the service quality improved by 26,73 points according to the Servqual scale, this difference was statistically validated with a Student's t test using a confidence interval of $95 \%$ and an experimental significance of 0,026 .

Keywords: quality of service, servqual, process improvement, study of times

\section{Resumo}

Esta pesquisa teve como objetivo implementar uma melhoria no fogo processo de cuidado para aumentar a qualidade do serviço prestado pelo voluntário Fire Company do Peru B 107 - Chimbote 2017. É utilizado um delineamento experimental com pré pré experimental teste teste e pós, sobre as pessoas em todos os processos de atendimento da empresa e como mostrado no fogo processo de cuidado, usando uma amostragem de conveniência não probabilística. $\mathrm{O}$ diagnóstico inicial mostrou um total de oito operações, um inspecao, um atraso e um transporte; da mesma maneira que calculamos uma média para a atenção de incêndios 54,62 minutos, 64,99 minutos de tempo normal e tempo padrão de 106,59 minutos de tempo; De igual modo a aplicação de Servqual questionário indicaram que as dimensões de qualidade de serviço eram a um nível médio. Subsequentemente, por meio de um diagrama causa - efectuar os necessários melhoramentos nos métodos de trabalho foi determinada através da optimização do tempo médio necessário para reduzir em 33,72 minutos, 40,12 minutos normal em tempo e o tempo padrão 65,80 minutos, representando uma redução de $61,74 \%$. Finalmente, concluiu-se que a qualidade do serviço melhorou em 26,73 pontos de acordo com a escala de Servqual, essa diferença foi validado estatisticamente com o teste t de Student usando um intervalo de confiança de $95 \%$ e um significado experimental de 0,026 .

Palavras-chave: qualidade do serviço, Servqual, melhoria do processo, estudo dos tempos

\section{Introducción}

En el mundo se produce miles de incendios de distintos tipos, por ejemplo según National Geographic existe una media de 100000 incendios forestales que queman entre 1,6 millones y 2 millones de hectáreas de terreno cada año, además de los miles de incendios en los hogares y otros lugares que ocurren causando grandes pérdidas humanas y materiales, a estos grandes desafíos se enfrentan los bomberos sobre todo el salvar las vidas de las personas cuando le acaecen estos accidentes. (National Geographic, 2014). En Europa aproximadamente 4000 personas fallecieron por motivo de incendios, y en España que es uno de los países con menor índice de muertes por causa de incendio en el año 2014 obtuvo un máximo de 116 personas fallecidas por causa de incendios, esto fue un incremento de $11.5 \%$ respecto al año anterior. (López, 2014) Se observa que a pesar de que España es uno de los países que tiene mejor índice de atención con respecto a los incendios, estos incrementaron en el 2014, teniendo todavía deficiencias al cubrir efectivamente los accidentes producidos por los incendios.

En Estados Unidos la cifra es más alarmante, pues anualmente 2500 personas mueren (estas muertes representan el $80 \%$ de las muertes por incendio) y 12600 sufren a causa de incendios en los hogares, además de las pérdidas en bienes materiales de aproximadamente 7300 millones de dólares por año. (Incendios en el hogar, Ready). Si bien las pérdidas materiales no podrían afectar mucho a Estados Unidos, las vidas que se pierden en estos incendios son los que realmente causan gran pena, pues la vida no tiene precio alguno, es por ello que el trabajo de atención de incendios en Estados unidos es más arduo, pues todavía tiene una cifra muy elevada de pérdidas en este tipo de accidentes. En Latinoamérica la situación es un poco más leve, debido a que las casas normalmente son de material noble y las llamas no se expanden rápido, a diferencia de los Estados unidos donde los hogares 
comúnmente son de madera, pues se menciona que no existen datos confiables de incendios en Latinoamérica, pero si hay informes sobre todo de incendios grandes pérdidas humanas y materiales. Por ejemplo en Argentina el incendio en la discoteca República Cromagnon allí 191 personas perdieron la vida, En Caracas, Venezuela 47 personas fallecieron a causa del incendio y en México murieron 20 personas y en Perú 29 personas. Lo que demuestra que las construcciones de ladrillo no son garantía de que nos protegeremos del incendio, es por ello la esperanza en que el trabajo del bombero sea excelente. (Nfpa Jla, 2017)

Todos estos casos internacionales de pérdidas humanas, materiales y también graves lesiones en los accidentados a causa de los incendios llaman a reflexión del gras reto que tienen los bomberos, y de lo fundamental que es para ellos y para todas las personas que cumplan su labor de forma efectiva siendo eficaz y eficiente a la vez, pues está en juego sobe todo la vida. Pero una situación aún más compleja se vive en Perú, pues más de 96000 incendios se produjeron en Perú el año 2016, se sabe de las grandes pérdidas que causan estos incendios, pues el Perú debido a sus limitaciones le afecta más estas pérdidas. (Gestión, 2016). Y específicamente en Ancash, en la ciudad de Nuevo Chimbote, se pasa por la misma situación pues los incendios afectan mucho a la población y a la ciudad. Debido al crecimiento poblacional se crean nuevos asentamientos o pueblos jóvenes donde la mayor parte de las viviendas son de materiales de fácil combustión, y esto hace que los incendios producidos en estos lugares sean aún más graves.

Se sustenta entonces que las causas de incendio tanto a nivel internacional, nacional y local se menciona que son debido a varios factores descritas al principio, y si bien la prevención y concientización de ser más responsables sobre estos temas de los incendios son importantes, el que ocurra uno de estos accidentes es inevitable pues puede ocurrir por distintos medios incluso por la naturaleza, por eso se ve la necesidad de mejorar la efectividad del trabajo de los bomberos. Así pues, se observa que esta realidad existen en todos las compañías de bomberos a nivel mundial, pues la atención que brindan los bomberos no es efectiva, ya que existen grandes pérdidas en estos tipos de accidentes y se asume que uno de los factores principales que resta la efectividad del trabajo de los bomberos, está relacionado con el proceso de atención. Por tanto para que los bomberos obtengan mejores resultados al cubrir los accidentes de tipo incendio, se necesita mejorar el proceso de atención, la cual ayudará a que los bomberos tengan mayor tiempo para el rescate de los afectados por el incendio

\section{Materiales y métodos}

El diseño de investigación fue pre experimental con pre test y post test; con dos grupos, esto debido a que primero se realizó una medición del nivel de servicio antes de la aplicación de la variable experimental, es decir de la implementación del proceso propuesto, luego se aplicó a otro grupo la variable experimental, es decir se puso en marcha el diseño del proceso de atención de incendios mejorado, para finalmente medir el nivel del servicio obtenido luego de la aplicación del proceso de atención propuesto. (Hernández y Batista, 2014).

La población estuvo conformada por personas afectadas por incendios ocurridos en la zona de Nuevo Chimbote, de los cuales se consideró una tamaño de muestra inicial de 11 afectados y posteriormente otra muestra de 11 personas afectadas sobre las cuales se utilizó los métodos de trabajo mejorados; así mismo se utilizó un muestreo no probabilístico por conveniencia.

En el caso de los resultados obtenidos para la variable de mejora del proceso, se empleó la metodología del estudio de trabajo: el uso de diagramas de análisis de procesos, diagrama de causa - efecto, toma de tiempos promedio, obtención del tiempo normal y del tiempo estándar. Para la calidad del servicio se utilizó el cuestionario SERVQUAL, el cual contaba con 32 preguntas y con una escala de medición del 1 al 5 para cada una de ellas.

Para el análisis inferencial, se aplicó inicialmente una prueba de normalidad con el test de ShapiroWilk para determinar si los resultados seguían una distribución normal y confirmar la fiabilidad de la prueba t de Student para la comprobación de hipótesis. En el caso de la prueba t de Student se procesó 
la información en el SPSS 22, utilizando la herramienta para muestras independientes. De la misma manera, se evaluó la relación de las variables a través del cálculo del Chi Cuadrado de Pearson utilizando una tabla de contingencia de $3 \times 2$.

\section{Resultados}

Para iniciar con el proceso de mejora de la calidad en el servicio de atención de incendios, por parte de la compañía voluntaria de bomberos B - 107, fue necesario identificar qué puntos generaban una tardía reacción e ineficiencia. Para identificar que procesos o inspecciones generaban estas demoras construyó un diagrama de flujo para poder tener un panorama más amplio respecto a las actividades que se dan solamente antes de salir de la compañía ya que los tiempos de recorrido desde la compañía hasta los puntos de incendios son tiempos incontrolables que no se van a poder considerar por su naturaleza errática. Para tener un mayor análisis se procedió a la elaboración de un cursograma que incluyó tiempos y distancias.

Emergencia

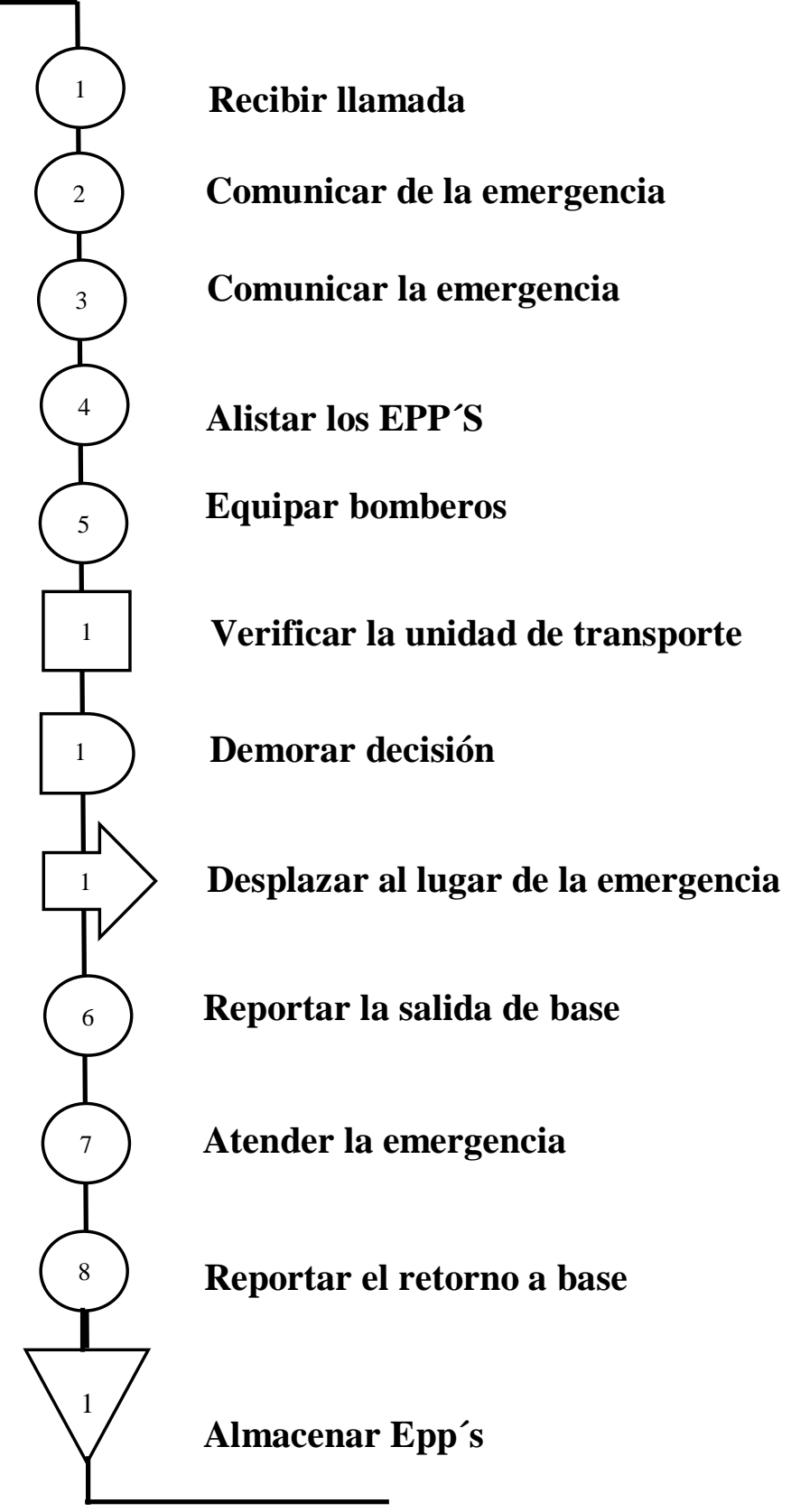

Atención de la Emergencia 
Figura 1. Diagrama operacional del proceso de atención de incendios de la compañía de bomberos de nuevo Chimbote B - 107

Fuente: Elaboración propia

En la Figura 1, se puede observar la muestra tomada en una emergencia de incendio en la Urb. Bruces, donde se observa tenemos ocho operaciones, dos inspecciones y un transporte, donde se detalla el tiempo de cada proceso y la distancia recorrida de la compañía hasta el lugar de la emergencia de incendio donde obtenemos el tiempo total empleado en una emergencia de incendio que atienda la compañía de bomberos B - 107. Luego se realizó un estudio que inició con un muestreo aleatorio del servicio de atención de incendios.

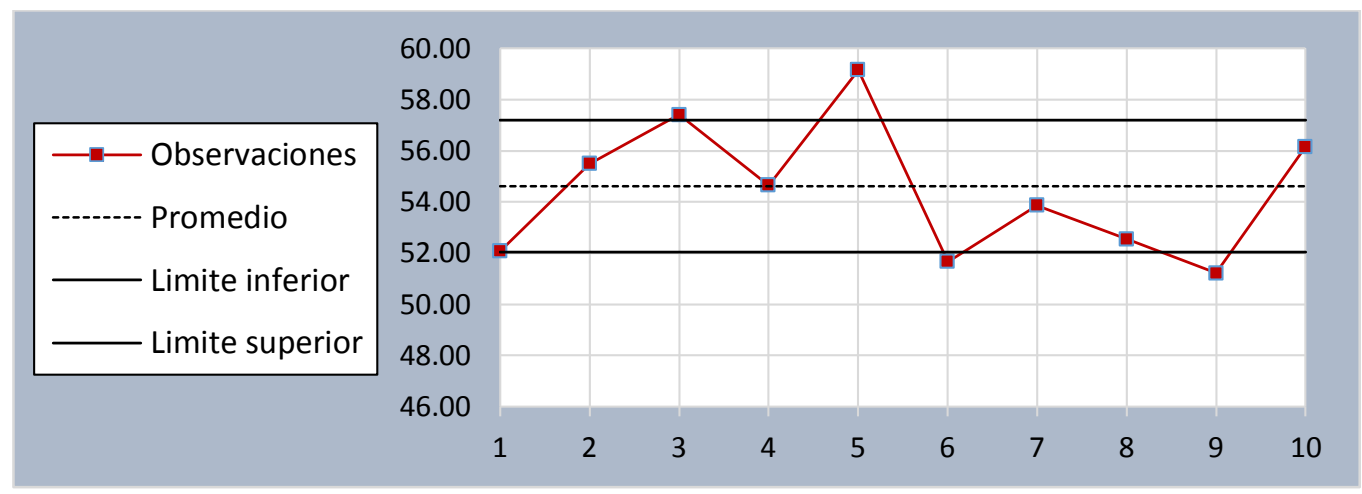

Figura 2. Tiempo promedio del proceso de atención de incendios de la compañía de bomberos de nuevo Chimbote B - 107

En la Figura 2, se analizó los tiempos observados con su promedio y una desviación estándar de 2,58 minutos. Habiendo determinado el tiempo promedio del servicio se procedió a convertirlo en un tiempo normal utilizando el Sistema de Valoración de Westinghouse, tal como se muestra en la siguiente tabla:

En la Tabla 3, se puede observar que los elementos tangibles están en un nivel medio con un 54,5\% ya que las personas consideran que los bomberos solo necesitan del vehículo cisterna y de su vestimenta (epp's) para atacar en la emergencia de incendio.

Tabla 1. Determinación del tiempo normal para el proceso de atención en el servicio de incendios

\begin{tabular}{llr}
\hline \multicolumn{1}{c}{ Factor } & \multicolumn{1}{c}{ Nivel } & Servicio de atención de incendio \\
\hline Habilidad & Excelente B2 & 0,08 \\
Esfuerzo & Excelente B2 & 0,08 \\
Condiciones & Buenas & 0,02 \\
Consistencia & Buena & 0,01 \\
1+ Factor de Valoración & 1,19 \\
\multicolumn{2}{c}{ Tiempo promedio } & 54,62 \\
\hline \multicolumn{2}{c}{ Tiempo normal } & $\mathbf{6 4 , 9 9}$ \\
\hline
\end{tabular}

En la Tabla 1, se observa que el tiempo normal para el servicio de atención en incendios corresponde a 64,99 minutos. Luego del tiempo normal se determinó el tiempo estándar con la siguiente tabla: 
Tabla 2. Determinación del tiempo estándar para el proceso de atención en el servicio de incendios

\begin{tabular}{cccccc}
\hline \multirow{2}{*}{ Frecuencia } & Porcentaje & $\begin{array}{c}\text { Porcentaje } \\
\text { válido }\end{array}$ & $\begin{array}{c}\text { Porcentaje } \\
\text { acumulado }\end{array}$ \\
\hline \multirow{3}{*}{ Válido } & & & & & \\
\cline { 2 - 6 } & Bajo & 2 & 18,2 & 18,2 & 18,2 \\
\cline { 2 - 6 } & Medio & 8 & 72,7 & 72,7 & 90,9 \\
\cline { 2 - 6 } & Alto & 1 & 9,1 & 9,1 & 100,0 \\
\cline { 2 - 7 } & Total & 11 & 100,0 & 100,0 & \\
\hline
\end{tabular}

Fuente: Cuestionario tabulado en SPSS

En la tabla 2, se observa que el tiempo estándar inicial del servicio de atención de incendios es de 106,59 minutos.

Para identificar el nivel de la calidad de servicio, se aplicó el cuestionario SERVQUAL para identificar cuáles son las deficiencias de la calidad de servicio de la compañía de bomberos voluntarios del Perú B - 107. Para ello el cuestionario se aplicó a 11 personas afectas a causa de los incendios dados en el distrito de Nuevo Chimbote.

Tabla 3. Elementos Tangibles para calidad del servicio en la atención de incendios

\begin{tabular}{clcccc}
\hline \multirow{4}{*}{ Válido } & Frecuencia & Porcentaje & $\begin{array}{c}\text { Porcentaje } \\
\text { válido }\end{array}$ & $\begin{array}{c}\text { Porcentaje } \\
\text { acumulado }\end{array}$ \\
\hline \multirow{3}{*}{} & Bajo & 1 & 9,1 & 9,1 & 9,1 \\
\cline { 2 - 6 } & Medio & 9 & 81,8 & 81,8 & 90,9 \\
\cline { 2 - 6 } & Alto & 1 & 9,1 & 9,1 & 100,0 \\
\cline { 2 - 6 } & Total & 11 & 100,0 & 100,0 & \\
\hline
\end{tabular}

En la Tabla 3, se puede observar que los elementos tangibles están en un nivel medio con un 54,5\% ya que las personas consideran que los bomberos solo necesitan del vehículo cisterna y de su vestimenta (epp's) para atacar en la emergencia de incendio.

Tabla 4. Nivel de capacidad de respuesta para calidad del servicio en la atención de incendios

\begin{tabular}{lr}
\hline \multicolumn{1}{c}{ Factor de Holgura } & Servicio de atención de incendio \\
\hline Fatiga & 4 \\
Necesidades personales & 5 \\
Uso de fuerza o energía muscular & 5 \\
Condiciones atmosféricas & 50 \\
& $1+\%$ Holgura \\
\multicolumn{1}{c}{ Tiempo normal } & 1,64 \\
\multicolumn{1}{c}{ Tiempo estándar } & 64,99 \\
\hline
\end{tabular}

Fuente: Cuestionario tabulado en SPSS

En la Tabla 4, se puedo determinar que el nivel de capacidad de respuesta está en un nivel medio con un $81,8 \%$; en este caso las personas afectadas consideraron que los bomberos contestan rápido ante una emergencia de incendio tratando de brindar la mejor calidad de servicio de atención. 
Tabla 5. Nivel de empatía para calidad del servicio en la atención de incendios

\begin{tabular}{clcccc}
\hline \multirow{2}{*}{ Válido } & Frecuencia & Porcentaje & $\begin{array}{c}\text { Porcentaje } \\
\text { válido }\end{array}$ & $\begin{array}{c}\text { Porcentaje } \\
\text { acumulado }\end{array}$ \\
\cline { 2 - 6 } & Bajo & 1 & 9,1 & 9,1 & 9,1 \\
\cline { 2 - 6 } & Medio & 9 & 81,8 & 81,8 & 90,9 \\
\cline { 2 - 6 } & Alto & 1 & 9,1 & 9,1 & 100,0 \\
\cline { 2 - 6 } & Total & 11 & 100,0 & 100,0 & \\
\hline
\end{tabular}

En la Tabla 5, se puede observar que el nivel de seguridad está en un nivel medio con un $72,7 \%$ las personas encuestadas manifiestan que la compañía de bombero les da seguridad al atender una emergencia de incendio porque están en cada emergencia solicitada.

Fuente: Cuestionario tabulado en SPSS

Tabla 6. Nivel de fiabilidad para calidad del servicio en la atención de incendios

\begin{tabular}{|c|c|c|c|c|c|}
\hline & & Frecuencia & Porcentaje & $\begin{array}{c}\text { Porcentaje } \\
\text { válido }\end{array}$ & $\begin{array}{l}\text { Porcentaje } \\
\text { acumulado }\end{array}$ \\
\hline \multirow{4}{*}{ Válido } & Bajo & 3 & 27,3 & 27,3 & 27,3 \\
\hline & Medio & 6 & 54,5 & 54,5 & 81,8 \\
\hline & Alto & 2 & 18,2 & 18,2 & 100,0 \\
\hline & Total & 11 & 100,0 & 100,0 & \\
\hline
\end{tabular}

En la Tabla 6, se puede observar que el nivel de empatía está en un nivel medio con un $81.8 \%$ las personas encuestadas manifiestan que a pesar de las largas distancias y demoras los bomberos llegan a atender la emergencia de incendio, mostrando un trato amable y cortes comprendiendo la situación que están pasando.

Tabla 7: Comparación de tareas con método inicial y mejorado en los procesos de atención.

\begin{tabular}{clcccc}
\hline & & Frecuencia & Porcentaje & $\begin{array}{c}\text { Porcentaje } \\
\text { válido }\end{array}$ & $\begin{array}{c}\text { Porcentaje } \\
\text { acumulado }\end{array}$ \\
\hline \multirow{4}{*}{ Válido } & Bajo & 2 & 18,2 & 18,2 & 18,2 \\
\cline { 2 - 6 } & Medio & 8 & 72,7 & 72,7 & 90,9 \\
\cline { 2 - 6 } & Alto & 1 & 9,1 & 9,1 & 100,0 \\
\cline { 2 - 6 } & Total & 11 & 100,0 & 100,0 & \\
\hline
\end{tabular}

Fuente: $\bar{C}$ uestionario tabulado en SPSS

En la Tabla 7, se puede observar que el nivel de empatía está en un nivel medio con un 72,7\% ya que las personas encuestadas manifiestan que el servicio que ofrecen los bomberos es confiable debido a que logran estar presentes y atacar la emergencia de incendio. 
Posteriormente se analizó las causas de los tiempos elevados en la atención de incendios de la compañía de bomberos.

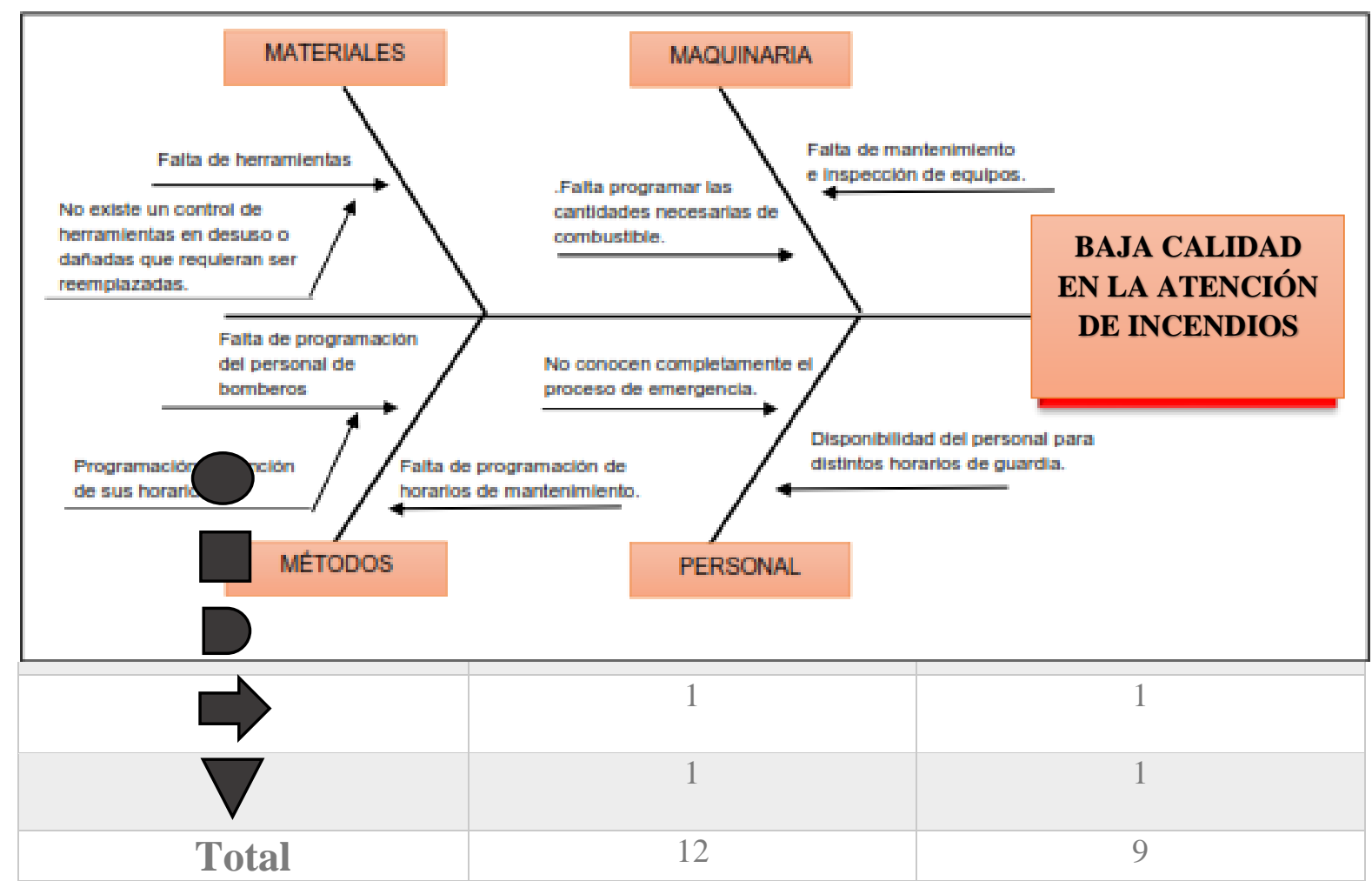

Figura 3. Diagrama causa-efecto de tiempos altos en la atención.

Mediante esta la espina de Ishikawa se plasman los problemas detectados en la compañía de bomberos voluntarios del Perú B - 107, en el cual me enfocare en las dos causas más críticas detectadas maquinaria y personal, ya que presentan deficiencias de mantenimiento de las unidades de rescate o salvamente y el personal bombero no disponible e apto para atender un incendio.

En función al Ishikawa se pudo observar oportunidades de mejora en la calidad de servicio realizando un cuadro resumen de operaciones.

Aplicando las mejoras diseñadas se volvió a medir los tiempos para determinar la optimización de los tiempos. 
Figura 4. Tiempo promedio mejorado del proceso de atención de incendios de la compañía de bomberos de nuevo Chimbote B - 107

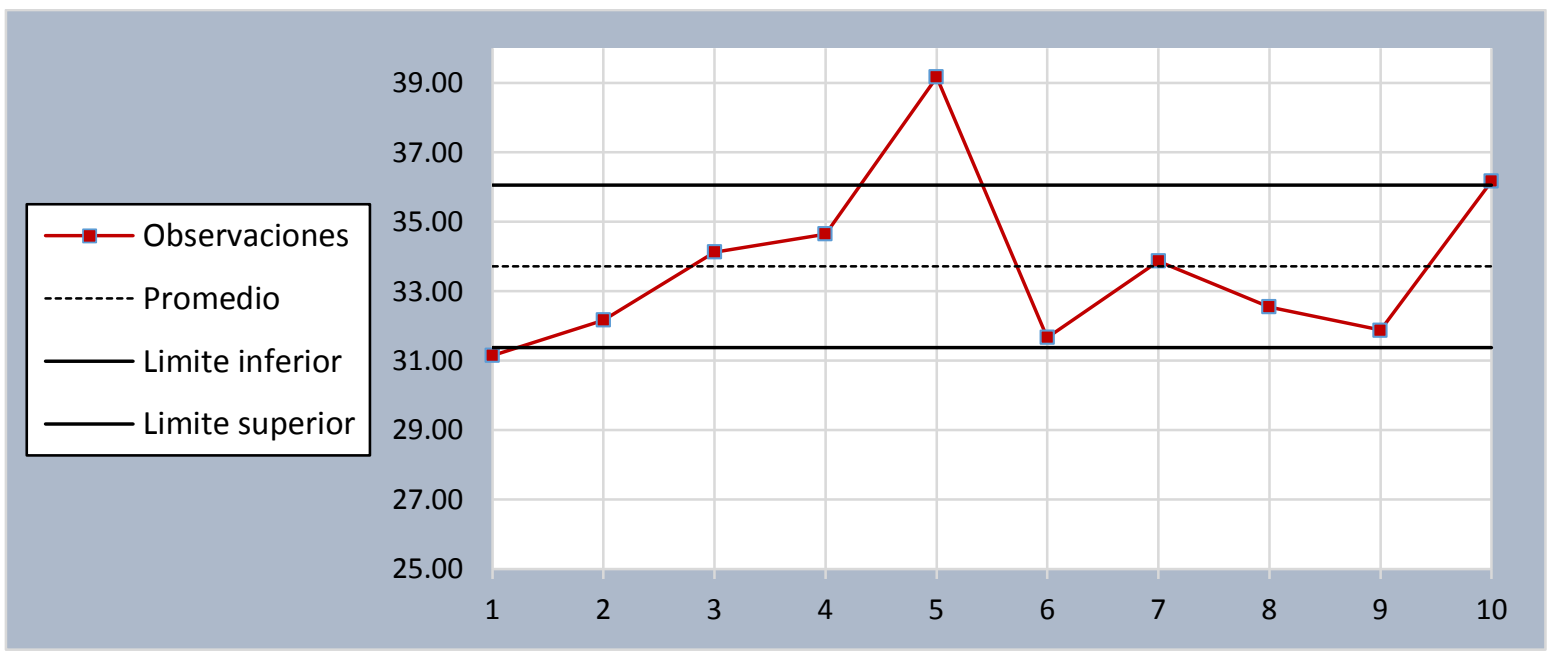

En la Figura 4, se analizó los tiempos mejorados obteniendo un promedio de 33,72 minutos y una desviación estándar de 2,34 minutos.

Tabla 8. Determinación del tiempo normal mejorado para el proceso de atención en el servicio de incendios

\begin{tabular}{lcr}
\hline Factor & Nivel & Servicio de atención de incendio \\
\hline Habilidad & Excelente B2 & 0,08 \\
Esfuerzo & Excelente B2 & 0,08 \\
Condiciones & Buenas & 0,02 \\
Consistencia & Buena & 0,01 \\
& 1+ Factor de Valoración & 1,19 \\
& Tiempo promedio & 33,72 \\
\hline & Tiempo normal & $\mathbf{4 0 , 1 2}$ \\
\hline
\end{tabular}

Habiendo determinado el tiempo promedio mejorado del servicio se procedió a convertirlo en un tiempo normal utilizando el Sistema de Valoración de Westinghouse.

Tabla 9. Determinación del tiempo estándar mejorado para el proceso de atención en el servicio de incendios

\section{Factor de Holgura}

Servicio de atención de incendio

Fatiga

4

Necesidades personales

Uso de fuerza o energía muscular

Condiciones atmosféricas

$$
1+\% \text { Holgura }
$$

Tiempo normal

En la Tabla 9, se puede observar que el tiempo normal mejorado es de 40,12 minutos 
Tabla 10. Determinación de la mejora en los tiempos del proceso de atención en el servicio de incendios

\begin{tabular}{cccc}
\hline & Inicial & Mejorado & Variación \\
\hline Tiempo estándar & 106,59 & 65,80 & $61,74 \%$
\end{tabular}

En la Tabla 10, se puede observar que el tiempo estándar mejorado es de 65,80 minutos

Con la aplicación del cuestionario SERVQUAL se logró cuantificar la percepción de los encuestados sobre la calidad del servicio en el proceso de atención de incendios; así mismo se aplicó el cuestionario luego de haber implementado las mejoras en los tiempos.

Tabla 11. Comparación del nivel de la calidad del servicio

\begin{tabular}{ccc}
\hline \multirow{2}{*}{ Muestra } & $\begin{array}{c}\text { Calidad del servicio } \\
\text { Puntuación SERVQUAL inicial }\end{array}$ & $\begin{array}{c}\text { Calidad del servicio } \\
\text { Puntuación SERVQUAL final }\end{array}$ \\
\hline 1 & 63 & 95 \\
2 & 87 & 78 \\
3 & 67 & 85 \\
4 & 99 & 129 \\
5 & 132 & 155 \\
6 & 85 & 69 \\
7 & 98 & 136 \\
8 & 96 & 151 \\
9 & 87 & 135 \\
10 & 94 & 142 \\
11 & 105 & 132 \\
\hline Media & $\mathbf{9 2 , 0 9}$ & $\mathbf{1 1 8 , 8 2}$ \\
\hline
\end{tabular}

En la Tabla 11 se puede observar que el tiempo estándar para el proceso de atención en el servicio de incendios se redujo en un $61,74 \%$ disminuyendo de 106,59 minutos a 65,80 minutos.

A pesar de que en un primer análisis se observa una mejora en los promedios de la percepción de la calidad del servicio, se sometió dichos resultados a las pruebas estadísticas correspondientes.

Tabla 12. Prueba de normalidad para valores del cuestionario SERVQUAL

\begin{tabular}{llccc}
\hline & \multirow{2}{*}{ Mejora de proceso } & \multicolumn{3}{c}{ Shapiro-Wilk } \\
& & Estadístico & gl & Sig. \\
\hline \multirow{2}{*}{ SERVQUAL } & Inicial &, 932 & 11 &, 429 \\
& Mejorado &, 875 & 11 &, 090 \\
\hline
\end{tabular}

Fuente: SPSS 22

En la Tabla 12, se observa que la calidad del servicio tuvo un puntaje promedio inicial de 92,09, utilizando el cuestionario SERVQUAL, y una puntuación final promedio de 118,92

A través del test de Shapiro-Wilk se pudo determinar que los resultados siguen una distribución normal debido a que los niveles de significancia son mayores al 0,05; en consecuencia si fue fiable comprobar la hipótesis con pruebas paramétricas como el t de Student. 
Tabla 13. Prueba t de Student para muestras independientes de la variable calidad del servicio

\begin{tabular}{|c|c|c|c|c|c|c|c|c|c|}
\hline & \multicolumn{2}{|c|}{$\begin{array}{l}\text { Prueba de } \\
\text { Levene de } \\
\text { calidad de } \\
\text { varianzas }\end{array}$} & \multicolumn{7}{|c|}{ prueba t para la igualdad de medias } \\
\hline & & & & & $\begin{array}{l}\text { Sig. } \\
\text { (bilat }\end{array}$ & Diferencia & $\begin{array}{l}\text { Diferencia } \\
\text { de error }\end{array}$ & $\begin{array}{r}95 \% \text { de in } \\
\text { confian } \\
\text { difer }\end{array}$ & $\begin{array}{l}\text { ervalo de } \\
\text { a de la } \\
\text { ncia }\end{array}$ \\
\hline & $\mathrm{F}$ & Sig. & $t$ & $\mathrm{gl}$ & eral) & de medias & estándar & Inferior & Superior \\
\hline $\begin{array}{l}\text { Se asumen } \\
\text { vaianzas } \\
\text { iguales }\end{array}$ & 6,783 & 017 & $-2,457$ & 20 &, 023 & $-26,72727$ & 10,87723 & $-49,41677$ & $-4,03778$ \\
\hline $\begin{array}{l}\text { No se } \\
\text { asumen } \\
\text { varianzas } \\
\text { iguales }\end{array}$ & & & $-2,457$ & 16,362 &, 026 & $-26,72727$ & 10,87723 & $-49,74456$ & $-3,70998$ \\
\hline
\end{tabular}

Fuente: SPSS 22

En la Tabla 13, se observa los resultados obtenidos del SPSS 22 considerando muestras independientes. Se inicia la tabla con la Prueba de Levene para determinar si las varianzas de los grupos son iguales, en ese caso el nivel de significancia es menor a 0,05 por lo cual se toma los resultados de la segunda fila al asumir varianzas desiguales entre los grupos.

En ese sentido se pudo determinar que las puntuaciones del cuestionario SERVQUAL tienen una diferencia en sus medias de $-26,72727$ la cual tiene un nivel de significancia de 0,026, aceptando la hipótesis de trabajo de que la mejora del proceso de atención de incendios incrementa la calidad del servicio brindado por la Compañía de Bomberos Voluntarios del Perú B 107.

Por otro lado, también se determinó la relación entre las variables de estudio, la cual puede analizarse en las tablas 15 y 16.

Tabla 14. Tabla de contingencia para las variables de mejora de proceso y nivel de calidad

\begin{tabular}{llccc}
\hline & & \multicolumn{3}{c}{ Nivel de Calidad } \\
\cline { 3 - 5 } & & Bajo & Medio & Alto \\
\cline { 2 - 5 } & & Recuento & Recuento & Recuento \\
\hline \multirow{2}{*}{ Mejora de proceso } & Inicial & 1 & 9 & 1 \\
\cline { 2 - 5 } & Mejorado & 0 & 4 & 7 \\
\hline
\end{tabular}

Fuente: SPSS 22 
Tabla 15. Prueba de Chi Cuadrado de Pearson para las variables de mejora de proceso y nivel de calidad del servicio

\begin{tabular}{llr}
\hline & & Nivel de Calidad \\
\hline Mejora de proceso & Chi-cuadrado & 7,423 \\
\cline { 2 - 3 } & gl & 2 \\
\cline { 2 - 3 } & Sig. &, 024 \\
\hline
\end{tabular}

En la Tabla 15, se elaboró una tabla para relacionar los niveles de calidad del servicio con los niveles de la mejora de procesos en los métodos de trabajo Compañía de Bomberos Voluntarios del Perú B 107.

Tabla 16. Prueba de Chi Cuadrado de Pearson para las variables de mejora de proceso y nivel de calidad del servicio

\begin{tabular}{llr}
\hline & & Nivel de Calidad \\
\hline Mejora de proceso & Chi-cuadrado & 7,423 \\
\cline { 2 - 3 } & gl & \multicolumn{2}{c}{2} \\
\cline { 2 - 3 } & Sig. & Nivel de Calidad \\
\hline \multirow{2}{*}{ Mejora de proceso } & \multicolumn{1}{c}{ Chi-cuadrado } & 7,423 \\
\cline { 2 - 3 } & gl & 2 \\
\cline { 2 - 3 } & Sig. &, 024 \\
\hline
\end{tabular}

En la Tabla 16, se estableció que las variables si tiene relación debido a que el nivel de significancia es menor al 0,05 .

\section{Discusión}

Para el diagnostico situacional, en este estudio, el diagrama causa -efecto se aplicó para analizar un problema previamente identificado y en el caso de la bibliografía revisada generalmente este se usa para identificar la causa raíz de una ocurrencia negativa. A pesar de ello la utilidad del instrumento fue de suma importancia ya que a través de él se puedo detectar que el proceso de atención de incendios presentaba tiempos elevados en su atención por una ineficiente secuencia de operaciones en sus métodos de trabajo. De la misma manera se hizo usos de símbolos no estándares para describir los procesos de trabajo, dichos símbolos son mencionados por NIEBEL (2009) y entre los cuales se encuentra la toma de decisiones; entonces, a pesar de no ser un símbolo estandarizado para un diagrama de análisis de operaciones fue importante resaltarlo porque a través de él se expresaba la continuidad o suspensión del proceso.

Otro punto importante fue el cálculo de los tiempos, sobre este punto se consideró que el número de observaciones requeridas se estableciera en función a la validez estadística de la toma tiempos, es decir que dependiendo de la desviación estándar y considerando una distribución t, por ser una muestra menor a 30, se decidió realizar solo 10 observaciones; esto discrepa con otros autores como BAYAS (2012), quien determinó su número de observaciones utilizando el criterio de General Electric donde ya se tiene una cantidad de mediciones preestablecidas dependiendo de la duración del tiempo ciclo en minutos.

Para el caso del cuestionario Servqual las dimensiones se encuentran estandarizadas por lo cual no se encuentra diferencia o contraste con otros investigadores, sin embargo si hay un contraste en lo que 
respecta a la escala de calificación; por ejemplo Pazmiño y Flor (2008) utilizaron siete alternativas para cada pregunta del cuestionario lo cual hace diferir con los puntajes obtenidos en la presente investigación, donde se utilizó una escala de Likert y con puntajes del 1al 5.

\section{Conclusiones}

Finalmente se pudo concluir que el proceso de atención de incendios en la Compañía de Bomberos Voluntarios del Perú B 107 - Chimbote 2017 contaba con un tiempo estándar inicial de 106,59 minutos y luego de la mejora de métodos de trabajo se pudo reducir en un 61,74\% llegando a 65,80 minutos; lo cual tuvo un impacto positivo en la percepción de la calidad del servicio, la cual fue medida con un cuestionario Servqual y su puntaje en promedio logró mejorar pasando de 92,09 a 118,82. Dicha diferencia, en los niveles de calidad, fue comprobaba a través de una prueba t de Student que estableció que si había diferencia estadística significativa entre ambas mediciones aceptándose la hipótesis de que una mejora en los métodos de trabajo si influye en la calidad del servicio de la Compañía de Bomberos Voluntarios del Perú B 107 - Chimbote.

\section{Referencias}

De Vicente y López (2012). Diseño de un modelo de riesgo integral de incendios forestales mediante técnicas multicriterio y su automatización en sistemas de información geográfica. (Tesis de Doctorado). Universidad Politécnica de Madrid, España. Recuperado de http://oa.upm.es/14779/1/FRANCISCO_JAVIER_DE_VICENTE_Y_LOPEZ_2.pdf

Euskalit (2017). Gestión y mejora de procesos. Recuperado de http://www.euskalit.net/pdf/folleto5.pdf

Faréz, A. \& SERRANO, V. (2014). Diseño De Un Sistema Organizacional De Gestión Por Procesos Aplicado En La Empresa Ferro Sarmiento. Tesis de pre grado). Recuperado de

Fernández Tabaré, (2003). Coeficientes de correlación lineal de Pearson. Recuperado de http://tabarefernandez.tripod.com/ficha20.pdf

García Bellido, R., González Such, J. y Jornet Meliá, J.(2010). Spss: Análisis De Fiabilidad Alfa De Cronbach. En Innovamide. Recuperado de http://www.uv.es/innomide/spss/SPSS/SPSS_0801B.pdf

Gestión (2016). Más de 96,000 incendios se registraron en el Perú este año. Recuperado de http://gestion.pe/tendencias/mas-96000-incendios-se-registraron-peru-este-ano-2173207

Hernández, S., Fernández, C. y Batista, L. (2014). Metodología de la investigación científica. México: Mc Graw - Hill.

López, S. (2015). Los incendios en viviendas provocan 116 muertes en 2014. El país. Recuperado de http://economia.elpais.com/economia/2015/01/16/vivienda/1421409021_239686.html

MONCADA, J. (2017). Documentación y estadísticas de incendios. Nfpajla. Recuperado de http://www.nfpajla.org/columnas/punto-de-vista/376-documentacion-y-estadisticas-deincendios

National Geographic (2010). Incendio. Recuperado de http://www.nationalgeographic.es/medioambiente/incendios

Pérez, R.( 2014). La calidad del servicio al cliente y su influencia en los resultados económicos y financieros de la empresa restaurante campestre sac - Chiclayo periodo enero a septiembre 2011 y 2012. (Tesis de Pre grado). Universidad Católica Santo Toribio De Mogrovejo, Perú. Recuperado de http://tesis.usat.edu.pe/bitstream/usat/203/1/TL_Perez_Rios_CynthiaKatterine.pdf 
Ramírez, P. (2014). Elaboración de un plan de emergencia y desarrollo e implementación del plan de contingencia, ante el riesgo de un incendio en el palacio del muy ilustre municipio de Guayaquil. (Tesis de Maestría). Universidad de Guayaquil, Ecuador. Recuperado de http://repositorio.ug.edu.ec/bitstream/redug/4806/1/Tesis\%20Maestria\%20Riesgos\%20y\% 20Desastres\%20JUAN\%20RAMIREZ.pdf

Ready. (2016). Incendios en el hogar. Recuperado de https://www.ready.gov/es/incendios-en-el-hogar

Rodríguez, M. (2011). Propuesta de un sistema de mejora continua para la reducción de mermas en una procesadora de vegetales en el departamento de Lima con el objetivo de aumentar su productividad y competitividad. (Tesis de pre grado). Universidad Peruana de Ciencias Aplicadas, Perú. Recuperado de

http://repositorioacademico.upc.edu.pe/upc/bitstream/10757/273503/1/CRodr\%C3\%ADgu ez.pdf

Sánchez, D. (2013). Indicadores: Seguimiento De La Gestión En La Empresa. Recuperado de https://www.aec.es/c/document library/get file?uuid=6680d3c1-4aeb-4f58-b787$\underline{8 \mathrm{~d} 2562 \mathrm{~cd} 9399 \& \text { groupId }=10128}$

Sigüenza, G. y Vicuña, P. (2015). Evaluación del Protocolo de Atención Prehospitalaria para emergencias médicas en politraumatismos atendidos por el Cuerpo de Bomberos y Sistema ECU 911, Azogues. (Tesis pre grado). Universidad de Cuenca, Ecuador. Recuperado de http://dspace.ucuenca.edu.ec/bitstream/123456789/23065/1/TESIS.pdf 